\title{
Clinical, cerebrospinal fluid, and neuroimaging findings in COVID-19 encephalopathy: a case series
}

\author{
Raphael L. Tuma ${ }^{1}$ (D) B Bruno F. Guedes ${ }^{1}$ (D) $\cdot$ Rafael Carra $^{1}$ (D) Bruno lepsen ${ }^{1}$ (D) Júlia Rodrigues $^{1}$ (D) \\ Antonio Edvan Camelo-Filho ${ }^{1}$ (D) - Gabriel Kubota ${ }^{1}$ (D) $\cdot$ Maíra Ferrari $^{1}$ (D) - Adalberto Studart-Neto ${ }^{1}$ (D) \\ Mariana Hiromi Oku ${ }^{1}$ (D) - Sara Terrim ${ }^{1}$ (D) - Cesar C. B. Lopes ${ }^{1}$ (D) $\cdot$ Carlos E. B. Passos Neto $^{1}$ (D) \\ Matheus D. Fiorentino ${ }^{1}$ (D) - Julia C. C. Souza ${ }^{1}$ (D) José Pedro S. Baima ${ }^{1}$ (D) Tomás Silva $^{1}$ (D) $\cdot$ lago Perissinotti ${ }^{1}$ (D) \\ Maria da Graça M. Martin ${ }^{2}$ (D) - Marcia Gonçalves ${ }^{1}$ (D) $\cdot$ Ida Fortini ${ }^{1}$ (D) - Jerusa Smid ${ }^{1}$ (D) Tarso Adoni $^{1}$ (D) \\ Leandro Lucato $^{2}$ (D) Ricardo Nitrini $^{1}$ (D) $\cdot$ Hélio Gomes $^{1}$ (D) $\cdot$ Luiz H. Castro $^{1}$ (B)
}

Received: 2 September 2020 / Accepted: 26 November 2020 / Published online: 7 January 2021

(C) Fondazione Società Italiana di Neurologia 2021

\begin{abstract}
Objective To describe the clinical, neurological, neuroimaging, and cerebrospinal fluid (CSF) findings associated with encephalopathy in patients admitted to a COVID-19 tertiary reference center.

Methods We retrospectively reviewed records of consecutive patients with COVID-19 evaluated by a consulting neurology team from March 30, 2020 through May 15, 2020.

Results Fifty-five patients with confirmed SARS-CoV-2 were included, 43 of whom showed encephalopathy, and were further divided into mild, moderate, and severe encephalopathy groups. Nineteen patients (44\%) had undergone mechanical ventilation and received intravenous sedatives. Eleven (26\%) patients were on dialysis. Laboratory markers of COVID-19 severity were very common in encephalopathy patients, but did not correlate with the severity of encephalopathy. Thirty-nine patients underwent neuroimaging studies, which showed mostly non-specific changes. One patient showed lesions possibly related to CNS demyelination. Four had suffered an acute stroke. SARS-CoV-2 was detected by RT-PCR in only one of 21 CSF samples. Two CSF samples showed elevated white blood cell count and all were negative for oligoclonal bands. In our case series, the severity of encephalopathy correlated with higher probability of death during hospitalization ( $\mathrm{OR}=5.5$ for each increment in the degree of encephalopathy, from absent (0) to mild (1), moderate (2), or severe (3), $p<0.001)$.

Conclusion In our consecutive series with 43 encephalopathy cases, neuroimaging and CSF analysis did not support the role of direct viral CNS invasion or CNS inflammation as the cause of encephalopathy.
\end{abstract}

Keywords COVID-19 $\cdot$ Encephalopathy $\cdot$ Cerebrospinal fluid $\cdot$ Critical care

Statistical analysis completed by Iago Perissinotti

Raphael L. Tuma

raphaeltuma96@gmail.com

1 Departamento de Neurologia, Hospital das Clínicas, Faculdade de Medicina, Universidade de São Paulo, Av. Dr. Enéas de Carvalho Aguiar, 255, $5^{\circ}$ andar, sala 5084 Cerqueira César, São Paulo, SP 05403-900, Brazil

2 Departamento de Neurorradiologia, Hospital das Clínicas, Faculdade de Medicina, Universidade de São Paulo, São Paulo, Brazil

\section{Introduction}

COVID-19 is a multisystem disease that usually targets the respiratory and cardiovascular systems. Neurological manifestations are common, occurring in up to $36.4 \%$ of cases [1]. The precise mechanism of neurologic involvement in COVID-19 remains incompletely understood, and may include neuroinvasion by severe acute respiratory syndrome coronavirus type 2 (SARS-CoV-2), cytokine storm, hypoxic, and vascular injury, as well as endothelial dysfunction [2]. Central nervous system invasion has not been consistently demonstrated.

Encephalopathy is a major complication of SARS-CoV-2 infection [3]. In previous studies, decreased consciousness was observed in 7.5-19.6\% of COVID-19 patients [1, 4, 5], 
and in $14.8-38.9 \%$ of severe cases $[1,5]$. Agitation and confusion occur commonly in patients with acute respiratory distress syndrome in COVID-19 [6]. In a previous series of consecutive COVID-19 patients evaluated by neurologists in a dedicated COVID-19 tertiary referral hospital, encephalopathy was the leading reason for a neurology consultation [7].

\section{Objective}

To describe the underlying conditions associated with encephalopathy in patients admitted to a COVID-19 reference center, we include systemic illness, intravenous sedative use, cerebrospinal fluid (CSF), and neuroimaging findings.

\section{Methods}

In March 2020, part of the Hospital das Clínicas da FMUSP was designated as a 900 bed (250 ICU) referral center dedicated to clinically suspected or confirmed COVID-19 patients. A neurology consult team consisting of seven neurologists and eight neurology residents (four PGY-2 and four PGY-3) provided on demand evaluations in the ICUs and wards. We retrospectively reviewed records of consecutive patients with COVID-19 evaluated by the neurology team from March 30, 2020 through May 15, 2020.

We selected cases with confirmed COVID-19, by positive SARS-CoV2 RT-PCR in nasopharyngeal swab or tracheal secretions or positive IgM or IgG serology by immunochromatography.

We classified patients in four groups according to the severity of the encephalopathy:

- No encephalopathy - fully awake, preserved sustained and basic attention, without neuropsychiatric disturbances or psychomotor slowing.

- Mild encephalopathy - awake or easily arousable patient, with preserved basic attention but impaired sustained attention. Patients with preserved sustained attention and level of consciousness, but presenting psychiatric, behavioral symptoms, or psychomotor slowing were also included in this group.

- Moderate encephalopathy - awake or easily arousable patient, with impaired basic and sustained attention.

- Severe encephalopathy - comatose patients or patients requiring vigorous stimuli to be aroused. Patients with severe psychomotor agitation were also included in this group.

Basic attention was defined as the ability to concentrate on a simple task, such as counting from 1 to 20 , reciting the months in direct order or forward-order digit span. Sustained or complex attention described the patient's ability to focus on a complex task that requires information processing, such as counting from 20 to 1 , reciting the months of the year backwards, or reverse-order digit span.

We did not include patients with pre-existing encephalopathy without significant worsening during the acute illness, encephalopathy occurring immediately after cardiopulmonary resuscitation, incomplete clinical or neurological evaluation, and fully awake patients with severe aphasia precluding attention evaluation. Patients still receiving intravenous sedatives were not excluded, since clinicians noted signs that warranted a neurology consultation despite sedation.

\section{Data collection}

We performed a systematic chart review for all enrolled patients collecting clinical data and neurological exam findings.

Outcome and additional neurological diagnoses established during follow-up were checked for all patients on June 1, 17 days after the evaluation period ended. Only laboratory tests collected within 3 days of the evaluation were included. CSF analysis included interleukin 6 (IL-6) quantification (electrochemiluminescence-Cobas ${ }^{\circledR}$ e 411, Roche), presence of oligoclonal bands (isoelectric focusing followed by immunoblotting), and CSF-SARS-CoV-2-RT-PCR (Abbot m2000 system Invitrogen Superscript IV and IDT primers and probes with 40 copies $/ \mathrm{mL}$ as the detection limit). Chest CT findings were classified as suggestive of viral pneumonia or not, based on radiology reports.

Brain computed tomography (CT), CT angiography, magnetic resonance imaging (MRI), and MRI angiography in encephalopathy patients were systematically reviewed by a trained neuroradiologist for acute changes. MRI was acquired in a $1.5 \mathrm{~T}$ GE scanner, including T1, T2, FLAIR, DWI, and susceptibility-weighted images and, when possible, FLAIR and T1-weighted images post gadolinium injection.

The study was approved by the hospital's ethics committee and is registered at http://plataformabrasil.saude.gov.br, certification number 33563420.0.0000.0068.

\section{Statistical analysis}

In the primary analysis, we applied multivariate ordinal logistic regression with the degree of encephalopathy as the endpoint. Predictors were selected based on previously described mortality predictors in COVID19 $[8,9]$ : age, gender, hypertension, diabetes, lymphocyte count, and C-reactive protein. We included two additional predictors to account for a potential contribution of neuroinflammation: CSF white blood cell count and CSF total protein. Intravenous sedation was also included as a predictor. In a secondary predetermined analysis, we applied logistic regression using encephalopathy degree as a predictor and mortality as an endpoint. 
Additionally, we performed an exploratory analysis. We applied multivariate ordinal logistic regression with the degree of encephalopathy as the endpoint and other laboratory parameters (platelets, BUN, d-dimer, presence of renal dysfunction, hypernatremia, and hyponatremia) as independent variables. Prior medical history was compared between groups using Mann-Whitney test.

\section{Results}

In the period between March 30 and May 15, 1720 patients were admitted to the COVID-19 dedicated facility, with 1263 confirmed SARS-CoV-2 infections. As of June 30, 812 of 1263 (64.3\%) COVID-19 patients had been discharged, 412 $(32.6 \%)$ had died, and $39(3.1 \%)$ were still in the hospital. The neurology team evaluated a total of 66 COVID-19 patients. Eleven patients were excluded. Fifty-five patients were included in the final analysis (Flowchart 1). All patients were also included in a previous study that evaluated reasons for neurology consultation in Hospital das Clínicas [7]. Detailed clinical and ancillary data of patients with encephalopathy were not provided in that report.

Patients were classified according to encephalopathy severity as follows: no encephalopathy, 12 cases; mild encephalopathy, 12 cases; moderate encephalopathy, 18 cases; severe encephalopathy, 13 cases.

Of the 43 encephalopathy cases, 33 consultations were requested due to acute altered mental state. In six cases, consultations were requested following abnormal brain $\mathrm{CT}$ results. Three consultations were requested to evaluate if a previously diagnosed neurological disease could contribute to the patient's current status and one for suspected acute focal deficit.
Of the 12 cases without encephalopathy, five consultations were requested to evaluate patients with neurological conditions preceding COVID-19 infection, which remained stable (two with previously operated tumors, one with myasthenia gravis, one with epilepsy, and one with a history of central venous thrombosis). Three were requested for headache, three for peripheral neuropathies, and one for somatoform disorder.

Demographic data is shown in Table 1, including the median number of days from initial respiratory symptoms to admission and days from admission to neurology consultation. Five patients (12\%) developed COVID-19 while already hospitalized for other conditions.

\section{Clinical data}

Arterial hypertension was the most common comorbidity in the encephalopathy group: 31 of 43 (72\%), followed by diabetes in $19(44 \%)$, renal disease in $11(26 \%)$, and smoking in $10(23 \%)$. No patient had HIV or AIDS. Fourteen $(33 \%)$ had a pre-existing neurologic condition (eight had a history of stroke, four of epilepsy, five had cognitive decline, and one had been diagnosed with primary CNS vasculitis).

Three of $32(9 \%)$ encephalopathy patients reported hyposmia and 16 of $36(44 \%)$ reported mental status changes, such as somnolence or confusion, prior to admission to the referral center. Three encephalopathy (7\%) patients reported seizures before admission, one of whom had a prior history of epilepsy. Three patients in the non-encephalopathy group had a history of epilepsy and presented breakthrough seizures before admission. Of the patients with confirmed COVID-19 and encephalopathy, chest $\mathrm{CT}$ was typical of viral infection in $37 / 39$ cases $(95 \%)$, compared to $8 / 12(67 \%)$ in the nonencephalopathy group.
Flowchart 1 From March 30 to May 15: 1720 patients admitted to the COVID dedicated facility; 1263 confirmed SARS-CoV-2 cases throughout the hospital; 66 COVID-19 patients received a neurology consultation, 43 with acute encephalopathy; 11 patients were excluded

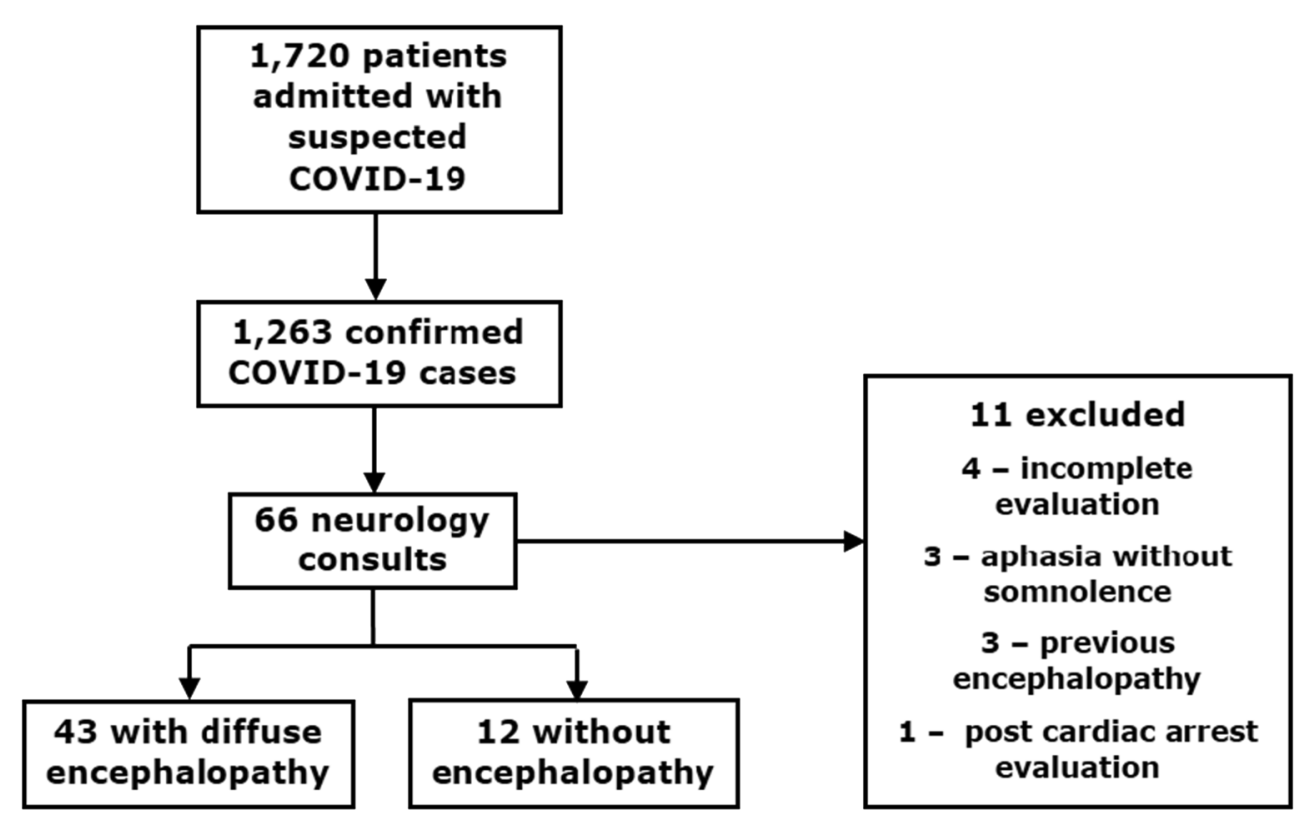




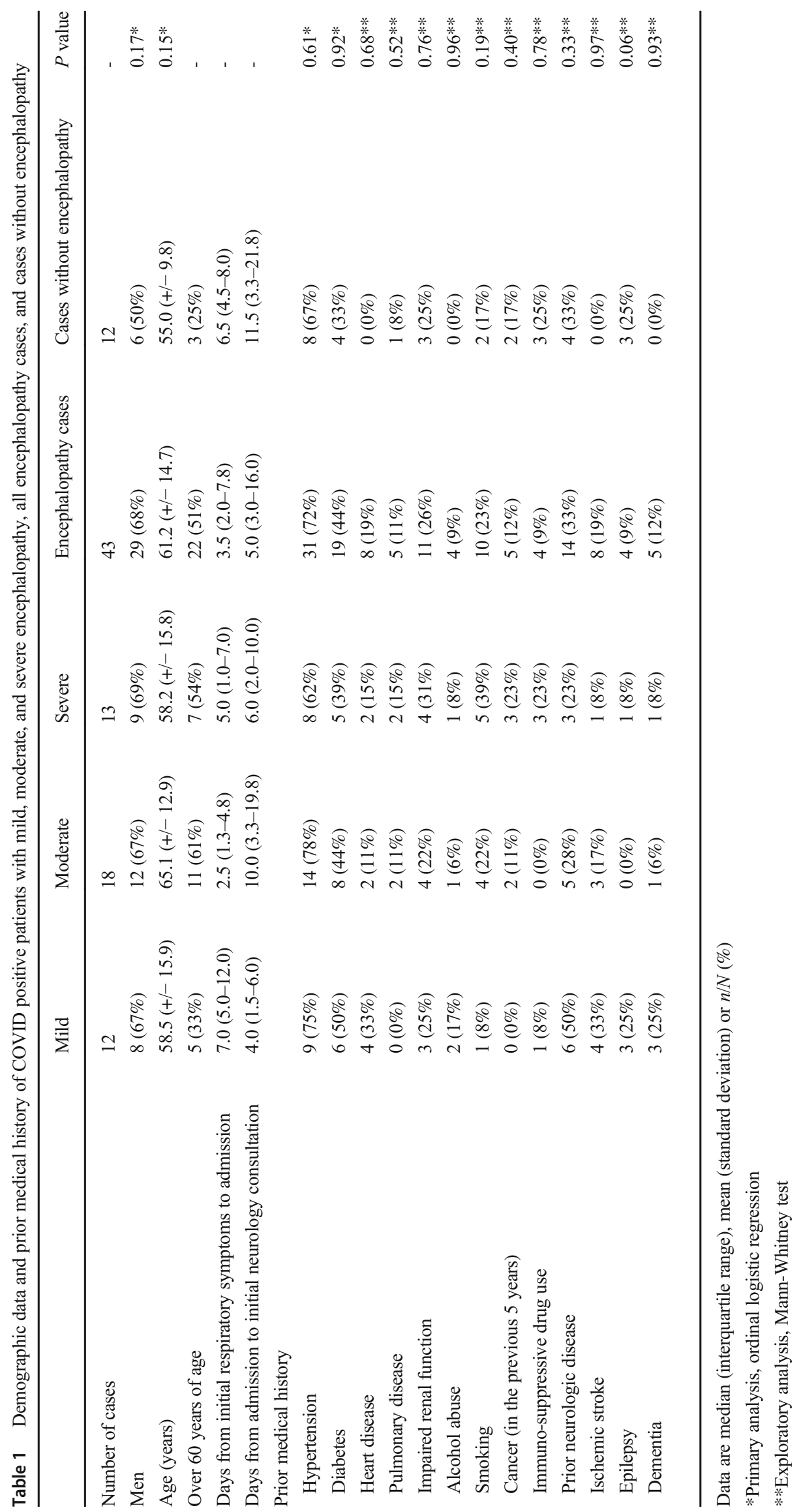




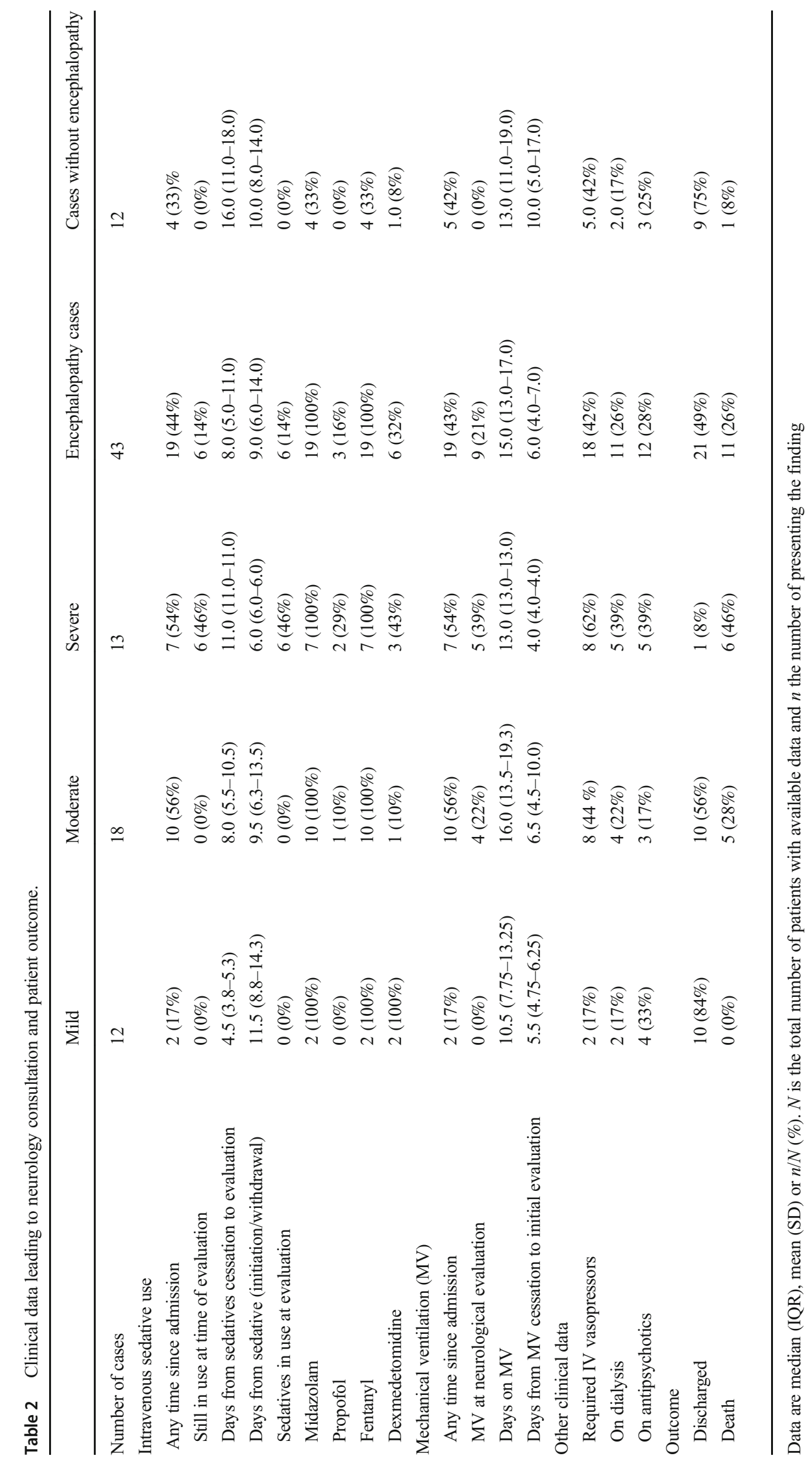




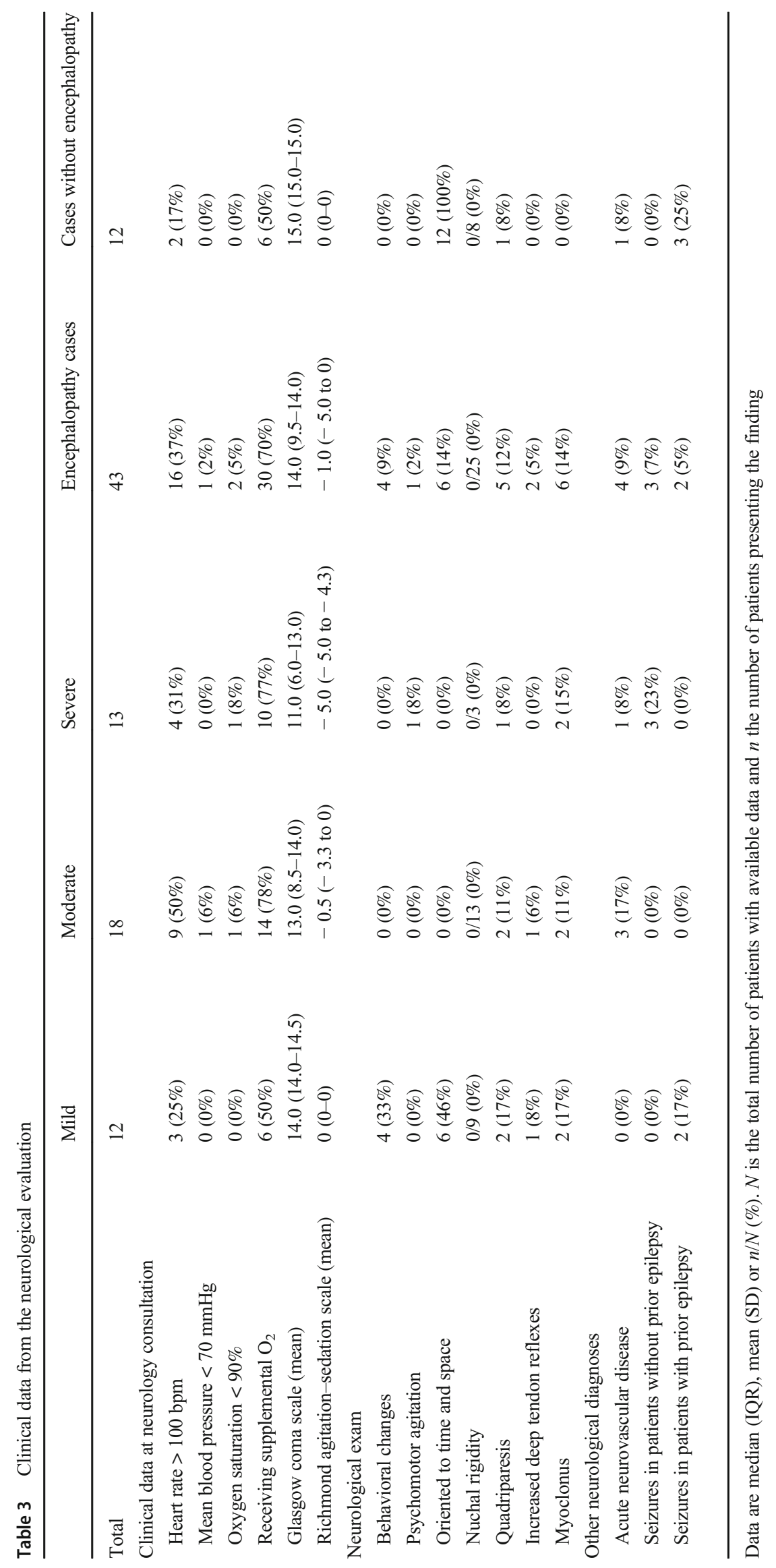




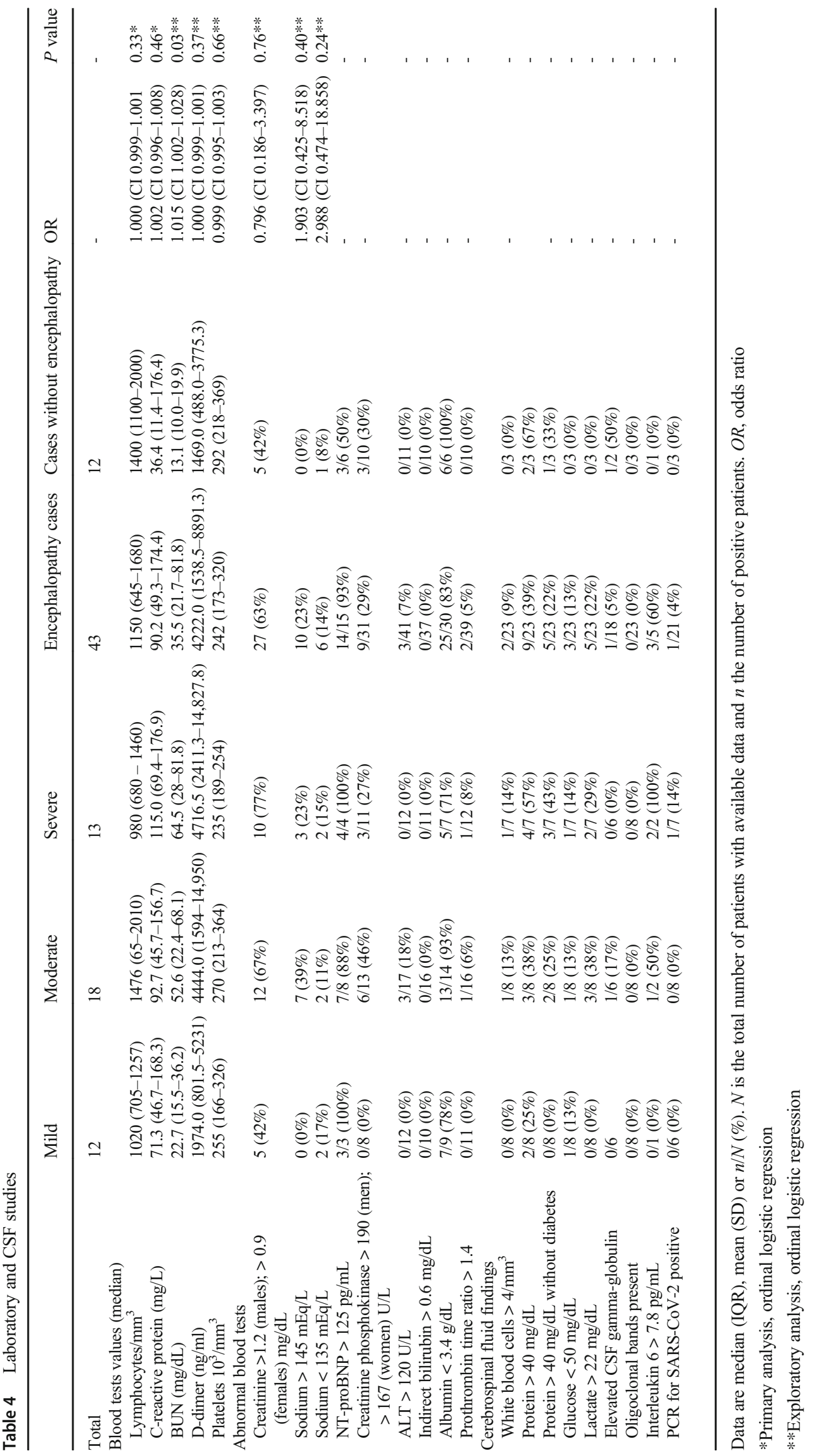


Mechanical ventilation, dialysis, vasopressor, and sedative use is shown in Table 2. During hospitalization, 19 (44\%) patients with encephalopathy received intravenous sedatives for mechanical ventilation, six (14\%) of whom were still under sedation on neurological evaluation. All these patients received midazolam and fentanyl. Three of the $19(16 \%)$ also received propofol, and six $(32 \%)$ also received dexmedetomidine. For the 13 patients no longer receiving intravenous sedatives on neurological evaluation, the median number of days from initiation of IV sedatives to IV sedative cessation was 9.0 (IQR 6.0-14.0), ranging from three to 42 days. Neurology evaluation occurred a median of 8.0 (IQR 5.0-11.0) days after sedation cessation (Table 2).

Table 3 presents neurological exam findings and clinical data obtained from chart review closest to the neurology consultation. Four (9\%) patients had preserved level of consciousness and attention, but were included in the mild encephalopathy group due to marked psychomotor slowing or acute psychiatric symptoms (psychosis and hypervigilance) without previous psychiatric disease.

Five patients had overt seizures; two of these had breakthrough seizures attributed to pre-existing epilepsy. Electroencephalogram could not be performed due to restrictions imposed by COVID- 19 .

Four patients, aged 45 to 59 years, had suffered acute ischemic strokes that could at least partially account for drowsiness. Three of these patients had focal deficits noted in the setting of slow neurologic recovery following IV sedative discontinuation. Of these four patients, two suffered malignant middle cerebral artery infarctions, one had an occipital and midbrain infarct, and the other one had a temporal-parietal infarct with hemorrhagic transformation (Table 3).

Laboratory test findings are presented in Table 4, including lymphocytes, platelets, creatinine, blood urea nitrogen (BUN), sodium, and C-reactive protein (CRP) for all patients. All encephalopathy patients had CRP above $5 \mathrm{mg} / \mathrm{L}$ (median 90.2; IQR 49.3-174.4 mg/L).

\section{Neuroimaging}

Thirty-nine of 43 encephalopathy patients underwent neuroimaging studies: brain CT 38, CT angiography 14, brain MRI nine, MRI angiography three. Neuroimaging was normal or showed only chronic changes in 29/38 (76\%) of CTs and 4/9 (44\%) of MRIs. Thirty of the 39 patients $(77 \%)$ did not present acute changes in neuroimaging studies.

Neuroimaging findings potentially associated with COVID19 were seen in five patients: acute ischemic stroke in the four described patients and one previously reported case [33] displayed multiple rounded T2/FLAIR hyperintensities possibly related to CNS demyelination. Four patients had acute brain imaging abnormalities probably not associated with COVID19: a pregnant woman with convexity subarachnoid hemorrhage (SAH), Wernicke's encephalopathy findings and pontine myelinolysis, a man with newly diagnosed brain metastasis from lung cancer, a transplant recipient with tacrolimusassociated posterior reversible encephalopathy syndrome, and a patient with a convexity subarachnoid hemorrhage alone. In the first SAH case, the finding could be attributed to Wernicke's encephalopathy or concurrent extrapontine myelinolysis. The second patient with convexity SAH did not have a history of trauma, coagulopathy, or vascular abnormalities on CT angiography. He underwent a brain $\mathrm{CT}$ after slow neurological recovery following a week of sedation and mechanical ventilation due to COVID-19 related acute respiratory syndrome, and did not display any other atypical findings that could relate to the $\mathrm{SAH}$. At the time of the evaluation, he displayed a c-reactive protein of $30.3 \mathrm{mg} / \mathrm{L}$ and d-dimer of $14,898 \mathrm{ng} / \mathrm{mL}$, with normal coagulation profile.

\section{Cerebrospinal fluid}

Lumbar puncture was performed in 23 encephalopathy patients and in three patients without encephalopathy who presented with headache. All three patients without encephalopathy showed normal CSF cell count and mildly elevated protein in two patients ( 42 and $48 \mathrm{mg} / \mathrm{dL}$ ).

Among encephalopathy patients, two (9\%) had an elevated CSF white blood cell (WBC) count. All 26 samples were negative for oligoclonal bands (Table 4).

Interleukin 6 was quantified in seven CSF samples from six patients (five encephalopathy patients and one nonencephalopathy patient). Interleukin 6 level values were in normal reference ranges $(<7.8 \mathrm{pg} / \mathrm{mL})$ in three patients, including one patient without encephalopathy $(2.13 \mathrm{pg} / \mathrm{mL})$, one patient with mild encephalopathy $(3.44 \mathrm{pg} / \mathrm{mL})$, and one with moderate encephalopathy $(7.32 \mathrm{pg} / \mathrm{mL})$. Three patients in the encephalopathy group had elevated IL-6 levels. One of these was case report \#2 (below), with moderate encephalopathy, and two IL-6 measures $(52.99 \mathrm{pg} / \mathrm{mL}$ and $25.32 \mathrm{pg} / \mathrm{mL}$ ). The two other patients had severe encephalopathy (19.57case report \#1, below—and $12.15 \mathrm{pg} / \mathrm{mL}$ ).

Case report \#1 A woman with peripheral eosinophilia, positive CSF SARS-CoV-2-RT-PCR, and elevated CSF IL-6SARS-CoV-2 was detected in the CSF of one patient (out of 21): a woman in her 50s who developed fever during hospitalization for acute aortic dissection complicated by renal failure requiring dialysis, blood eosinophilia, disseminated candidiasis, and central venous catheter-related bloodstream infection. A nasal swab test was positive for SARS-CoV-2. Two days after onset of fever, she developed encephalopathy. A lumbar puncture, obtained 10 days after onset of fever, revealed 15 cells $/ \mathrm{mm} 3$ (38\% lymphocytes, $8 \%$ monocytes, $22 \%$ neutrophils, $31 \%$ eosinophils, and $1 \%$ macrophages), protein of $54 \mathrm{mg} / \mathrm{dL}$, interleukin 6 of $19.57 \mathrm{pg} / \mathrm{mL}$ (cut-off 
$7.8 \mathrm{pg} / \mathrm{mL}$ ), and a positive CSF SARS-COV-RT-PCR. A peripheral blood cell count also revealed elevated leukocytes count with $48 \%$ eosinophils. Brain CT was normal, and MRI was not obtained.

Case report \#2 A man presents with EBV meningoencephalitis, CSF pleocytosis, and elevated CSF IL-6. A man over the age of 70 with positive nasal swab SARS-CoV-2-RT-PCR was transferred to our center due to encephalopathy 28 days after respiratory symptom onset.

Brain CT was normal. CSF analysis revealed $256 / \mathrm{mm}^{3}$ cells (90\% mononuclear cells), a total protein $115 \mathrm{mg} / \mathrm{dL}$, glucose $46 \mathrm{mg} / \mathrm{dL}$, lactate $25.2 \mathrm{mg} / \mathrm{dL}$, and IL-6 $52.99 \mathrm{pg} /$ $\mathrm{mL}$. A CSF multiplex PCR assay detected EBV and CSFSARS-CoV-2-PCR was negative. Additional lumbar punctures were performed 3 and 7 days after the initial exam, and showed persistent CSF pleocytosis, elevated IL-6 (25.32 pg/ $\mathrm{mL}$ ) and elevated total protein, but negative EBV-PCR. The patient fully recovered after 1 month.

\section{Statistical analysis}

None of the variables showed a correlation with the severity of encephalopathy in the primary ordinal logistic regression analysis. On the secondary predetermined analysis, the degree of encephalopathy correlated with higher probability of death during hospitalization; OR of 5.5 for each increment in the degree of encephalopathy $(p<0.001)$.

On exploratory analysis of prior medical history and other laboratory tests, only BUN correlated with the severity of encephalopathy $(\mathrm{OR}=1.015$, CI: $1.002-1.028, p=0.027)$. $P$ values were not corrected for multiple comparisons.

\section{Discussion}

We report the findings of encephalopathy in 43 patients from 66 neurology consults in 1263 COVID-19 patients. The presentation of encephalopathy ranged from behavioral changes and mild attention deficit to severely impaired consciousness. Its degree correlated with mortality

Our series represents cases in the COVID-19 dedicated center presenting with more severe neurological manifestations, or those in which clinicians could not attribute symptoms to systemic dysfunctions alone. These cases would represent the sample of patients most likely to present neurological disease caused by direct central nervous system viral invasion. However, evidence of neuroinvasion and neuroinflammation were largely absent. Although clinical factors, including renal impairment and prolonged sedative use were highly prevalent in moderate and severe encephalopathy cases, many patients displayed encephalopathy without clear metabolic dysfunction or sedative use, especially in mild encephalopathy cases. The only consistent findings in these patients were laboratory signs of systemic inflammation.

Neurological examination showed few additional findings other than encephalopathy. Some patients presented myoclonus, attributed to toxic and metabolic disorders. In two patients, abnormal movements noted on neurological exam suggested focal seizures. Only one patient in this series presented severe psychomotor agitation on examination. In other patients, agitation had been controlled with antipsychotics and intravenous sedatives by the time of the neurology evaluation.

The moderate encephalopathy group was the largest group in our series, comprising patients seen later in the disease course, many of whom had spent prolonged periods under sedation and mechanical ventilation. These patients were easily arousable, but had severely compromised level of attention, in some cases unable to engage in basic attention testing.

Although encephalopathy is a common finding in COVID-19 inpatients, the precise underlying mechanisms remain incompletely understood. Case reports or small-case series that evaluated SARS-CoV-2 RNA detection in CSF samples showed conflicting results: while some studies detected viral RNA with RTPCR or next-generation sequencing [10-14], other studies reported negative findings [15-20]. Generalizability of findings is possibly limited by publication bias. Our study included $24 \mathrm{CSF}$ SARS-CoV-2-RT-PCR tests. We found only one SARS-CoV-2positive sample, which may have resulted from shedding of viral genetic material from plasma to CSF in a patient with damaged blood-brain barrier. Our findings suggest that SARS-CoV-2 RNA detection rates in encephalopathy cases may be lower than expected from case reports, but consistent with other larger case series of neurologic disease [6,21-23]. Other studies demonstrated the presence of anti-SARS-CoV-2 antibodies in the CSF of patients with neurologic disease and negative CSF-RT-PCR [19, 20], suggesting that immunological assays may be superior to molecular testing to demonstrate SARS-CoV-2 CNS involvement. In other infectious diseases, such as Lyme neuroborreliosis, neurosyphilis, West Nile virus encephalitis and Japanese encephalitis, immunological assays appear to be superior to molecular testing in blood or CSF, which may be related to low pathogen loads or transient CNS infections [24].

Alternatively, encephalopathy may be caused by CNS immune-mediated processes without viral invasion. Our series is in agreement with larger studies showing that CSF cell and biochemistry counts are largely normal in COVID-19 patients with neurological symptoms [5, 6, 19, 21]. However, elevated CSF WBC, protein levels, and positive oligoclonal bands were described in case reports [3]. Oligoclonal bands were absent in all 24 CSF samples in our series. Only two patients ( $8.3 \%$ of cases) showed increased CSF WBC. In both cases, alternative explanations not related to SARS-CoV-2 CNS infection (case reports \#1 and \#2) were postulated.

Cytokine storm is a potential major cause of acute respiratory distress syndrome and multiple organ dysfunction in 
COVID-19 patients [25]. Cytokine serum levels, including IL-1, IL-6, and TNF- $\alpha$, correlate with systemic COVID-19 severity $[26,27]$. Recent studies demonstrated elevated CSF IL-6 levels in patients with encephalopathy, negative CSF SARS-CoV-2-RT-PCR, and normal CSF cell count, suggesting a potential role of IL- 6 as a biomarker in these patients $[23,28]$. We found markedly elevated IL-6 levels in two patients with known inflammatory CNS disease, both of which were possibly not COVID-related-in case report \#1, elevated IL-6 was probably the result of viral meningoencephalitis; the patient in case report 2\#, had bloodstream infection, disseminated candidiasis and elevated levels of eosinophils in both blood and CSF, which implied systemic inflammation from other causes compromised the blood-brain barrier.

Although most COVID-19 neuroimaging studies evaluated patients with acute cerebrovascular disease, suspected myelopathy and miscellaneous neurologic syndromes, neuroimaging was requested to evaluate mental status changes in $37-$ $73 \%$ of patients [29-32]. In our series, the majority of patients had brain CTs or MRIs, most of which did not display acute changes. Infarcts (10.3\% of neuroimaging studies) were the most common acute findings, in keeping with other retrospective studies [31, 32]. Only one case, reported elsewhere [33], had acute demyelinating-like lesions potentially related to COVID-19. In other COVID-19 patients, neuroimaging findings could be explained by other neurological diseases; however, their association with COVID-19 cannot be completely ruled out. In our series, we did not observe other previously described neuroimaging findings, such as punctate hemorrhagic foci, cortical swelling, and leptomeningeal enhancement [34]. Since only a few patients in this series underwent MRI, these findings could be missed on brain $\mathrm{CT}$.

\section{Conclusion}

In our series, encephalopathy in COVID-19 manifested in various degrees of severity which were associated with increased mortality and may be influenced by factors such as sedative drug use, organ system dysfunction, and systemic inflammation. Head CT showed mainly non-specific changes, and MRI may be preferable, though not easily obtainable in critically ill patients, to document more subtle findings related to encephalopathy in COVID-19 patients. CSF analysis in our series did not detect viral genetic material or inflammatory changes, suggestive of CNS viral invasion or immunemediated processes, in the majority of encephalopathy cases. Future studies should clarify the role of cytokines, CSFSARS-CoV-2 immunology, and other inflammatory biomarkers, particularly in mild and moderate cases of COVID19 associated encephalopathy.
Authors' contributions Raphael de Luca e Tuma: Conceptualized the paper, collected data, participated in consensus meetings, analyzed data and wrote the initial draft; Bruno Fukelmann Guedes: Participated in consensus meetings, analyzed data and wrote the initial draft; Rafael Bernhart Carra: Participated in consensus meetings, collected data data and wrote the initial draft; Bruno Diógenes Iepsen: Participated in consensus meetings, collected data data and wrote the initial draft; Júlia Chartouni Rodrigues: Participated in consensus meetings, collected data data and wrote the initial draft; Antonio Edvan Camelo Filho: Data collection and analysis; Gabriel Taricani Kubota: Data collection and analysis; Maíra Medeiros Honorato Ferrari: Data collection and analysis; Adalberto Studart-Neto: Data collection and analysis; Mariana Hiromi Manoel Oku: Data collection and analysis; Sara Terrim: Data collection and analysis; Cesar Castello Branco Lopes: Data collection and analysis; Carlos Eduardo Borges Passos Neto: Data collection and analysis; Matheus Dalben Fiorentino: Data collection and analysis; Julia Carvalhinho Carlos De Souza: Data collection and analysis; José Pedro Soares Baima: Data collection and analysis; Tomás Fraga Ferreira Da Silva: Data collection and analysis; Iago Navas Perissinotti: Completed the statistical analysis; Maria da Graça Martin: Reviewed the neuroimaging studies; Marcia Rubia Rodrigues Gonçalves: Participated in consensus meetings and reviewed the manuscript for intellectual content; Ida Fortini: Participated in consensus meetings and reviewed the manuscript for intellectual content; Jerusa Smid: Participated in consensus meetings and reviewed the manuscript for intellectual content; Tarso Adoni: Participated in consensus meetings and reviewed the manuscript for intellectual content; Leandro Tavares Lucatto: Reviewed the neuroimaging studies; Ricardo Nitrini: Participated in consensus meetings, wrote and reviewed the manuscript for intellectual content and supervised the study; Helio Rodrigues Gomes: Participated in consensus meetings, wrote and reviewed the manuscript for intellectual content and supervised the study; Luiz Henrique Martins Castro: Participated in consensus meetings, wrote and reviewed the manuscript for intellectual content and supervised the study. The corresponding author takes full responsibility for the data, the analyses and interpretation, and the conduct of the research. He has full access to all of the data and has the right to publish any and all data separate and apart from any sponsor.

Compliance with ethical standards As stated in the Methods section of the manuscript, this study was approved by the hospital's ethics committee.

Conflict of interest The authors declare that they have no conflict of interest.

\section{References}

1. Mao L, Jin H, Wang M et al (2020) Neurologic manifestations of hospitalized patients with coronavirus disease 2019 in Wuhan, China. JAMA Neurol. https://doi.org/10.1001/jamaneurol.2020. 1127

2. Wu Y, Xu X, Chen Z et al (2020) Nervous system involvement after infection with COVID-19 and other coronaviruses. Brain Behav Immun 87:18-22. https://doi.org/10.1016/j.bbi.2020.03.031

3. Garg RK, Paliwal VK, Gupta A (2020) Encephalopathy in patients with COVID-19: A review. J Med Virol. https://doi.org/10.1002/ jmv.26207

4. Cummings MJ, Baldwin MR, Abrams D et al (2020) Epidemiology, clinical course, and outcomes of critically ill adults with COVID-19 in New York City: a prospective cohort study. Lancet Lond Engl 395:1763-1770. https://doi.org/10.1016/ S0140-6736(20)31189-2 
5. Romero-Sánchez CM, Díaz-Maroto I, Fernández-Díaz E et al (2020) Neurologic manifestations in hospitalized patients with COVID-19: The ALBACOVID registry. Neurology. https://doi. org/10.1212/WNL.0000000000009937

6. Helms J, Kremer S, Merdji $\mathrm{H}$ et al (2020) Neurologic features in severe SARS-CoV-2 infection. N Engl J Med 382:2268-2270. https://doi.org/10.1056/NEJMc2008597

7. Studart-Neto A, Guedes BF, Tuma R d L e et al (2020) Neurological consultations and diagnoses in a large, dedicated COVID-19 university hospital. Arq Neuropsiquiatr. https://doi. org/10.1590/0004-282x20200089

8. Jordan RE, Adab P, Cheng KK (2020) Covid-19: risk factors for severe disease and death. BMJ 368:m1198. https://doi.org/10.1136/ bmj.m1198

9. Stokes EK, Zambrano LD, Anderson KN et al (2020) Coronavirus disease 2019 case surveillance - United States, January 22-May 30, 2020. MMWR Morb Mortal Wkly Rep 69:759-765. https://doi. org/10.15585/mmwr.mm6924e2

10. Moriguchi T, Harii N, Goto J et al (2020) A first case of meningitis/ encephalitis associated with SARS-Coronavirus-2. Int J Infect Dis IJID Off Publ Int Soc Infect Dis 94:55-58. https://doi.org/10.1016/ j.ijid.2020.03.062

11. Huang YH, Jiang D, Huang JT (2020) SARS-CoV-2 detected in cerebrospinal fluid by PCR in a case of COVID-19 encephalitis. Brain Behav Immun 87:149. https://doi.org/10.1016/j.bbi.2020.05. 012

12. Domingues RB, Mendes-Correa MC, Vilela de Moura Leite FB et al (2020) First case Of SAR-Coronavirus-2 sequencing in the cerebrospinal fluid of a patient with suspected CNS demyelinating disease. https://doi.org/10.21203/rs.3.rs-31801/v1

13. Sun T, Guan J Novel coronavirus and the central nervous system. Eur J Neurol. https://doi.org/10.1111/ene.14227

14. Zhou L, Zhang M, Wang J, Gao J (2020) Sars-Cov-2: underestimated damage to nervous system. Travel Med Infect Dis:101642. https://doi.org/10.1016/j.tmaid.2020.101642

15. Al Saiegh F, Ghosh R, Leibold A et al (2020) Status of SARS-CoV2 in cerebrospinal fluid of patients with COVID-19 and stroke. J Neurol Neurosurg Psychiatry 91:846-848. https://doi.org/10.1136/ jnnp-2020-323522

16. Juliao Caamaño DS, Alonso Beato R (2020) Facial diplegia, a possible atypical variant of Guillain-Barré Syndrome as a rare neurological complication of SARS-CoV-2. J Clin Neurosci Off J Neurosurg Soc Australas 77:230-232. https://doi.org/10.1016/j. jocn.2020.05.016

17. Kilinc D, van de Pasch S, Doets AY et al (2020) Guillain-Barré syndrome after SARS-CoV-2 infection. Eur J Neurol. https://doi. org/10.1111/ene. 14398

18. Sancho-Saldaña A, Lambea-Gil Á, Liesa JLC et al (2020) GuillainBarré syndrome associated with leptomeningeal enhancement following SARS-CoV-2 infection. Clin Med Lond Engl 20:e93-e94. https://doi.org/10.7861/clinmed.2020-0213

19. Andriuta D, Roger P-A, Thibault W et al (2020) COVID-19 encephalopathy: detection of antibodies against SARS-CoV-2 in CSF. J Neurol. https://doi.org/10.1007/s00415-020-09975-1

20. Benameur K, Agarwal A, Auld SC et al (2020) Encephalopathy and encephalitis associated with cerebrospinal fluid cytokine alterations and coronavirus disease, Atlanta, Georgia, USA, 2020. Emerg Infect Dis 26. https://doi.org/10.3201/eid2609.202122
21. Neumann B, Schmidbauer ML, Dimitriadis K et al (2020) Cerebrospinal fluid findings in COVID-19 patients with neurological symptoms. J Neurol Sci 418:117090

22. Destras G, Bal A, Escuret V et al (2020) Systematic SARS-CoV-2 screening in cerebrospinal fluid during the COVID-19 pandemic. Lancet Microbe 1:e149

23. Alexopoulos H, Magira E, Bitzogli K et al (2020) Anti-SARSCoV-2 antibodies in the CSF, blood-brain barrier dysfunction, and neurological outcome: studies in 8 stuporous and comatose patients. Neurol Neuroimmunol Neuroinflamm 7

24. He T, Kaplan S, Kamboj M, Tang Y-W (2016) Laboratory diagnosis of central nervous system infection. Curr Infect Dis Rep 18:35. https://doi.org/10.1007/s1 1908-016-0545-6

25. Coperchini F, Chiovato L, Croce L et al (2020) The cytokine storm in COVID-19: an overview of the involvement of the chemokine/ chemokine-receptor system. Cytokine Growth Factor Rev 53:2532. https://doi.org/10.1016/j.cytogfr.2020.05.003

26. Ragab D, Salah Eldin H, Taeimah M et al (2020) The COVID-19 cytokine storm; what we know so far. Front Immunol 11:1446. https://doi.org/10.3389/fimmu.2020.01446

27. Wang J, Jiang M, Chen X, Montaner LJ (2020) Cytokine storm and leukocyte changes in mild versus severe SARS-CoV-2 infection: review of 3939 COVID-19 patients in China and emerging pathogenesis and therapy concepts. J Leukoc Biol 108:17-41. https://doi. org/10.1002/JLB.3COVR0520-272R

28. Farhadian S, Glick LR, Vogels CBF et al (2020) Acute encephalopathy with elevated CSF inflammatory markers as the initial presentation of COVID-19. BMC Neurol 20:248. https://doi.org/10. 1186/s12883-020-01812-2

29. Jain R, Young M, Dogra S et al (2020) COVID-19 related neuroimaging findings: a signal of thromboembolic complications and a strong prognostic marker of poor patient outcome. J Neurol Sci 414:116923. https://doi.org/10.1016/j.jns.2020.116923

30. Kremer S, Lersy F, de Sèze J, et al (2020) Brain MRI findings in severe COVID-19: a retrospective observational study. Radiology 202222. https://doi.org/10.1148/radiol.2020202222

31. Mahammedi A, Saba L, Vagal A et al (2020) Imaging in neurological disease of hospitalized COVID-19 patients: an Italian multicenter retrospective observational study. Radiology:201933. https:// doi.org/10.1148/radiol.2020201933

32. Radmanesh A, Raz E, Zan E et al (2020) Brain imaging use and findings in COVID-19: a single academic center experience in the epicenter of disease in the United States. AJNR Am J Neuroradiol 41:1179-1183. https://doi.org/10.3174/ajnr.A6610

33. Lopes CCB, Brucki SMD, Passos Neto CEB et al (2020) Acute disseminated encephalomyelitis in COVID-19: presentation of two cases and review of the literature. Arq Neuropsiquiatr. https://doi. org/10.1590/0004-282X20200186

34. Kandemirli SG, Dogan L, Sarikaya ZT et al (2020) Brain MRI findings in patients in the intensive care unit with COVID-19 infection. Radiology:201697. https://doi.org/10.1148/radiol. 2020201697

Publisher's note Springer Nature remains neutral with regard to jurisdictional claims in published maps and institutional affiliations. 